elSSN 2444-7986

DOI: http://dx.doi.org/10.14201/orl201672.14234

Caso clínico

\title{
CEFALEA RINÓGENA POR CONTACTO MUCOSO CON SOLUCIÓN QUIRÚRGICA. DESCRIPCIÓN DE UN CASO
}

\author{
Rhinologic headache caused by mucosal contact with a surgical \\ solution. A case report
}

Ignacio ALCALÁ-RUEDA; José Miguel VILLACAMPA-AUBÁ; Álvaro SÁNCHEZ-BARRUECO; Carlos CENJOR-ESPAÑOL

Hospital Universitario Fundación Jiménez Díaz. Servicio de Otorrinolaringología. Madrid. España.

Correspondencia: ignacio.alcala@quironsalud.es

Fecha de recepción: 7 de abril de 2016

Fecha de aceptación: 27 de abril de 2016

Fecha de Publicación: 10 de mayo de 2016

Conflicto de intereses: Los autores declaran no tener conflictos de intereses

Imágenes: Los autores declaran haber obtenido las imágenes con el permiso de los pacientes

Política de derechos y autoarchivo: se permite el autoarchivo de la versión post-print (SHERPA/RoMEO)

Licencia CC BY-NC-ND. Licencia Creative Commons Atribución-NoComercial-SinDerivar 4.0 Internacional

(C) Universidad de Salamanca. Su comercialización está sujeta al permiso del editor

RESUMEN

Introducción y objetivo: La cefalea rinógena por contacto mucoso nasal es un cuadro de difícil definición con buenos resultados quirúrgicos. Descripción: Presentamos el caso de un varón de 31 años con criterios de cefalea rinógena. Decidimos manejo quirúrgico. Discusión: La cefalea rinógena se encuentra dentro del grupo de «cefaleas ocasionadas por alteraciones en la mucosa nasal, cornetes o tabique nasal». No existe acuerdo en cuanto a su etiopatogenia por igual proporción de contacto mucoso nasal en pacientes con y sin dolor facial. Sin embargo se ha evidenciado resolución del dolor con tratamiento quirúrgico. Conclusiones: Pese a la existencia de mejoría con tratamiento quirúrgico, todavía quedan dudas en cuanto a las causas de esta entidad.

PALABRAS CLAVE cefaleas secundarias; dolor facial; mucosa nasal; tabique nasal, sinusitis; enfermedades nasales

SUMMARY Introduction and objective: Rhinologic headache caused by mucosal contact is a difficult definition condition with good response to surgical approach. Description: We present a case of a 31 year-old male with rhinologic headache criteria. We decided to offer a surgical approach, successfully. Discussion: Rhinologic headache is included in the group of «Headache caused by disorders of the nasal mucosa, turbinates or septum». There is no agreement about its cause because the prevalence of mucosal nasal contact is similar in patients with and without facial pain. There is evidence in the resolution of facial pain with a surgical approach. Conclusions: Despite the evidence of success in the surgical approach, some doubts still persist about the causes of this entity.

KEYWORDS

headache disorders, secondary; facial pain; nasal mucosa; nasal septum; sinusitis; nose diseases

(c) Ediciones Universidad de Salamanca / CC BY-NC-ND [117]

Rev. ORL, 2016, 7, 2, pp. 117-120 


\section{INTRODUCCIÓN}

La cefalea rinógena es una entidad cuya definición siempre ha sido polémica. Por una lado podría englobar cualquier algia producida por una ocupación nasosinusal y, por otro lado, más específica, se ha definido como una cefalea hemifacial relacionada con síntomas congestivos en pacientes con contactos de las mucosas a nivel nasal (sobre todo entre el cornete medio y el septo) $[1,2]$. La prevalencia de algias faciales en pacientes con clínica rinógena es aproximadamente de un $25 \%$, aunque la causa más frecuente de dolor facial no es esta [3]. Sin embargo, la incidencia de puntos de contacto intermucosos es frecuente tanto en pacientes sintomáticos como en asintomáticos [3-5].

Existen unos criterios diagnósticos definidos por la International Headache Society (IHS) para diagnosticarla (Tabla 1) [1, 4].

El tratamiento de la cefalea rinógena debe de ser primero médico, relegando la cirugía a los casos que no respondan a tratamiento médico [5].

Partiendo de un paciente con sospecha de cefalea rinógena, discutiremos su tratamiento y evolución comparándolo con la bibliografía existente.

Tabla 1. Criterios diagnósticos de «cefalea ocasionada por alteraciones en la mucosa nasal, cornetes o tabique nasal» según la Headache Classification Subcommittee of the International Headache Society.

A. Cualquier cefalea que cumpla el criterio en $\mathrm{C}$.

B. Evidencia clínica, radiológica o endoscópica de proceso inflamatorio o hipertrófico dentro de la cavidad nasal. (Ejemplos: Concha bullosa o espolón septal)

C. Evidencia de causa mostrada por al menos dos de los siguientes:

1. Cefalea desarrollada en relación temporal con la aparición de la lesión intranasal.

2. Cefalea que mejora o empeora de forma significativa en paralelo a una mejoría o empeoramiento de la lesión nasal.

3. Cefalea que mejora de forma significativa tras anestesia local de la mucosa en la región de la lesión.

4. Cefalea ipsilateral al sitio de la lesión nasal.

D. Cefalea que no se explica mejor por otro diagnóstico del ICH.

\section{DESCRIPCIÓN}

Presentamos el caso de un varón de 31 años, trabajador social de profesión, sin antecedentes médicos de interés y no fumador que fue derivado a nuestra consulta por cefaleas periorbitarias en relación con exposición a irritantes nasales, especialmente tras permanecer expuesto a tabaco de forma pasiva y en ambientes cerrados con aire acondicionado. Los episodios presentaban una duración media de 7 a 14 días, con ausencia de resolución con antiinflamatorios no esteroideos habituales. A su vez refería rinorrea no purulenta y alguna epistaxis autolimitada independiente de estos episodios. Ningún dato de la anamnesis apuntaba a un origen neurológico de dicha cefalea. En la exploración física se apreciaba una mucosa nasal edematosa y una desviación del tabique nasal obstructiva derecha en el área IV de Cottle. La exploración neurológica era compatible con la normalidad. Debido a la intensa clínica del paciente, se decidió solicitar una tomografía computarizada (TC) de senos paranasales para una mejor filiación del cuadro y una valoración por el servicio de Alergología. En el TC encontramos un leve engrosamiento mucoperióstico del seno frontal derecho y en el receso alveolar maxilar ipsilateral. Se constató la presencia de un espolón óseo septal derecho en contacto con cornete medio ipsilateral que se mostraba hipoplásico. El resto de las estructuras no presentaban alteraciones significativas (Figuras 1 y 2 ).

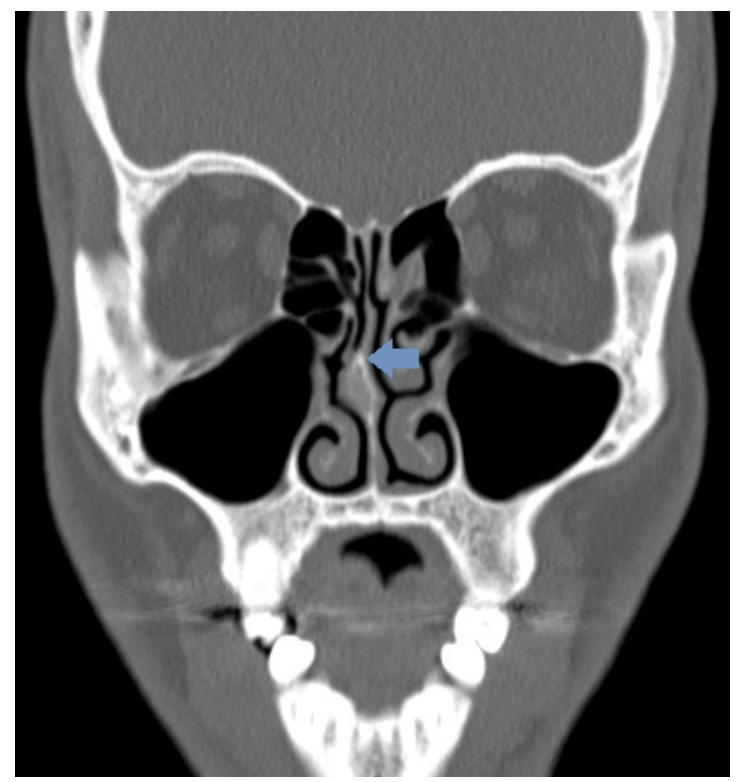

Figura 1. Corte coronal de TC nasosinusal. Se aprecia espolón óseo septal derecho contactando con cornete hipoplásico derecho. 


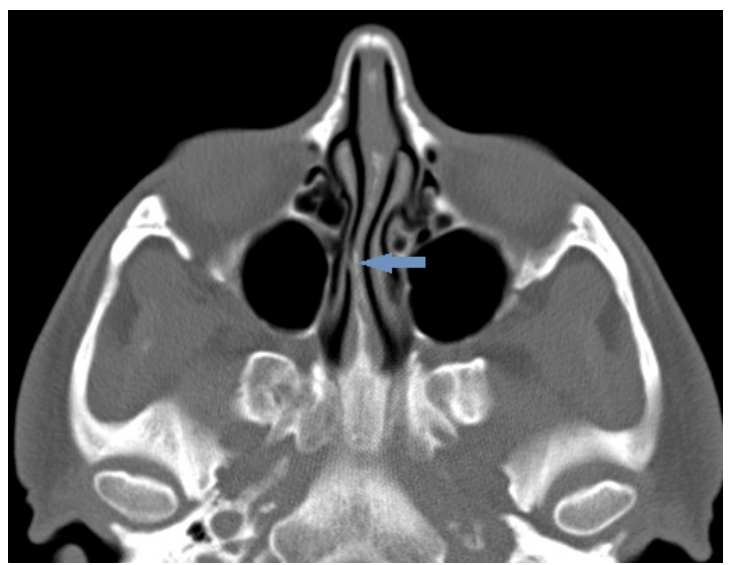

Figura 2. Corte axial de TC nasosinusal. Espolón óseo que condiciona un espacio respiratorio estrecho a nivel de meato medio. Se aprecia contacto mucoso entre septum nasal y cornete medio hipoplásico derecho.

En el servicio de Alergología se filió la causa de la rinorrea como una rinitis perenne vasomotora. En el estudio alérgico se encontró únicamente sensibilización a polen de Lolium.

Ante la falta de respuesta al tratamiento conservador y sospechando una cefalea rinógena debido a la ausencia de indicios que apuntaran en otro sentido, se decidió analizar la respuesta ante el test de aplicación de anestésico tópico nasal. Este presentó un resultado positivo dado que, tras la aplicación tópica de lidocaína y adrenalina en la zona sospechosa, se confirmó una mejoría sintomática inmediata. Ante dicho resultado se ofreció al paciente la posibilidad de realización de septoplastia con turbinoplastia mediante abordaje microscópico según técnica de Cottle modificada, con intención de eliminar a su vez el contacto mucoso descrito. El paciente aceptó y firmó el consentimiento informado, procediendo entonces a la intervención.

El postoperatorio cursó de forma favorable, sin incidencias. En el postoperatorio inmediato se constató la ausencia de cefalea y, tras cuatro años de seguimiento, no ha presentado recidiva de las mismas.

\section{DISCUSIÓN}

Según la tercera edición de la International Clasification of Headache Disorders by the International Headache Society [4], el cuadro descrito se encuentra dentro de la categoría de «cefaleas ocasionadas por alteraciones en la mucosa nasal, cornetes o tabique nasal». A pesar de ello la validez de esta entidad es cuestionada por algunos autores quienes la consideran una forma de dolor neuropático sin fundamento anatómico [1, 2]. El término de "cefalea rinógena» debe diferenciarse del de «cefalea sinusal», caracterizada esta última por congestión nasal y rinorrea purulenta, con aumento de dolor a la palpación de los senos paranasales y en relación a procesos sinusales agudos o crónicos [1,4]. Otros autores sin embargo, diferencian únicamente entre cefalea rinógena y no rinógena [3].

Aquellos autores -entre ellos Stammberger y Wolf-que defienden la existencia de un factor anatómico causante relacionan su etiopatogenia con la liberación de "sustancia $P$ » y otros neuropéptidos por contacto de las mucosas, que actúan sobre el nervio trigémino, desencadenando dolor y otras alteraciones sensitivas en función de la susceptibilidad del paciente [1, $2,5,6]$. La conexión de las fibras nerviosas cervicales con las raíces aferentes del nervio trigémino podría explicar además la aparición de cefaleas en casi cualquier punto entre la cabeza y el cuello [7].

Por otra parte, otros autores dudan de la importancia de este contacto mucoso en la génesis de esta cefalea, alegando la elevada incidencia de estos contactos en pacientes asintomáticos, incluso operados (técnica de sinequias controladas), así como un posible efecto placebo como la causa de mejoría tras tratamiento quirúrgico, señalando la ausencia de ensayos clínicos adecuados para la evaluación de este hecho [1, 2]. Abu-Bakra, et al. [7] estudiaron 973 pacientes con diagnóstico de rinosinusitis, divididos en cohortes en función de la existencia de dolor facial, encontrando en ambas un $4 \%$ de puntos de contacto mucoso intranasales, no existiendo diferencia por tanto entre ambos. Otro estudio conducido por los mismos autores consistió en la aplicación de «sustancia $P$ », adrenalina, presión local manual y placebo, demostrando la ausencia de dolor facial tras la aplicación de los mismos, con la presencia únicamente de picor nasal y estornudos tras la aplicación de «sustancia P» [8].

Estos autores argumentan además la mejoría sólo parcial de las cefaleas tras tratamiento quirúrgico y la ausencia de seguimiento más allá de unos meses en los pacientes intervenidos en esta patología, lo que no es aplicable a nuestra experiencia [8, 9].

Por el contrario, existe bibliografía que respalda la mejoría de la clínica de cefalea tras el cese del contacto mucoso. Kunachack [10] 
realizó un estudio en el que lateralizaba el cornete medio en consulta con un abordaje mínimamente invasivo y bajo anestesia local en pacientes con cefalea y evidencia endoscópica de contacto de mucosa nasal. De este modo, la cefalea mejoraba de manera muy notable y mantenida, con un seguimiento medio de 50 meses tras el procedimiento. De hecho, según la literatura publicada en los últimos años, y recogida por Mehle [1], algunas series demuestran la mejoría clínica tras la cirugía hasta en el 54 al $92 \%$ de los pacientes.

Mariotti [11] va un paso más allá, apuntando no sólo a la disminución del contacto mucoso como causa de la mejoría tras cirugía, sino también a la mejora de la ventilación nasosinusal. De la misma forma, hace hincapié en que, a pesar de la mejoría tras el tratamiento quirúrgico, es imprescindible la creación de algunas directrices de consenso sobre el diagnóstico y el tratamiento, probablemente mediante un estudio multicéntrico controlado.

Por tanto, en base a la bibliografía, debe buscarse la minimización del contacto de las mucosas, existiendo un acuerdo generalizado en comenzar con el tratamiento médico mediante corticosteroide nasal, descongestionantes nasales y lavados nasales con preparados salinos. Sólo en caso de fracaso del mismo, se planteará el tratamiento quirúrgico $[1,2,5]$. La prueba preoperatoria con anestesia tópica parece aceptada como de gran utilidad como indicador predictor del éxito de la cirugía.

\section{CONCLUSIONES}

La cefalea rinógena persiste como una entidad de difícil definición. En su diagnóstico, una vez descartada otro tipo de cefalea, parece relevante el test de anestesia tópica con adrenalina, como diagnóstico y predictor del éxito quirúrgico. El tratamiento de inicio debe ser médico, relegando el quirúrgico a un segundo tiempo. No existe acuerdo en cuanto a su etiopatogenia ni en cuanto a la explicación de la mejoría tras el tratamiento quirúrgico.

\section{BIBLIOGRAFÍA}

1. Mehle ME. What do we know about rhinogenic headache? The otolaryngologist's challenge. Otolaryngol Clin North Am. 2014;47(2):255-64.
2. Harrison L, Jones NS. Intranasal contact points as a cause of facial pain or headache: a systematic review. Clin Otolaryngol. 2013;38(1):8-22.

3. Agius AM, Sama A. Rhinogenic and nonrhinogenic headaches. Curr Opin Otolaryngol Head Neck Surg. 2015;23(1):15-20.

4. Headache Classification Committee of the International Headache S. The International Classification of Headache Disorders, 3rd edition (beta version). Cephalalgia. 2013;33(9):629-808.

5. Sadeghi M, Saedi B, Ghaderi Y. Endoscopic management of contact point headache in patients resistant to medical treatment. Indian J Otolaryngol Head Neck Surg. 2013;65(Suppl 2):415-20.

6. Stammberger H, Wolf G. Headaches and sinus disease: the endoscopic approach. Ann Otol Rhinol Laryngol Suppl. 1988;134:3-23.

7. Puig CM, Driscoll CLW, Kern EB. Sluder's Sphenopalatine Ganglion Neuralgia;Treatment with $88 \%$ Phenol. Am J Rhinol. 1998;12(2):113-8.

8. Abu-Bakra M, Jones NS. Prevalence of nasal mucosal contact points in patients with facial pain compared with patients without facial pain. J Laryngol Otol. 2001;115(8):629-32.

9. Abu-Bakra M, Jones NS. Does stimulation of nasal mucosa cause referred pain to the face? Clin Otolaryngol Allied Sci. 2001;26(5):430-2.

10. Kunachak S. Middle Turbinate Lateralization: A Simple Treatment for Rhinologic Headache. Laryngoscope. 2002;112(5):870-2.

11. Mariotti LJ, Setliff RC, Ghaderi M, Voth S. Patient history and CT findings in predicting surgical outcomes for patients with rhinogenic headache. Ear Nose Throat J 2009;88:926-9. 